\title{
Looking at a Large-Scale Clinical Studies from a Local
}

\section{Perspective}

\section{Todic MR*}

Department of Pharmacology, University of Sarajevo, Bosnia and Herzegovina

*Corresponding author: Maida Rakanovic Todic, Department of Pharmacology and

Toxicology Faculty of Medicine, University of Sarajevo, Bosnia and Herzegovina, Tel: +38733217540; Email: maida.todic@mf.unsa.ba

\section{Editorial}

Volume 2 Issue 6

Received Date: November 18, 2019

Published Date: November 27, 2019

DOI: $10.23880 /$ jqhe- 16000146

\section{Editorial}

Medical science perceives randomized clinical trials (RCT) as the gold standard in the development of new drugs and source of providing valuable new treatment options. RCT results are indisputably used in the production of therapeutic guidelines and healthcare policy making. The health authorities of most developed countries are aware of the benefits of large-scale clinical trials. Thus, their websites often include sections dedicated to those. Issues they typically address include the application process for clinical trials or the potential benefits and risks for the participants. It seems that potential benefits for patients participating in large-scale clinical trials may vary greatly depending of the level of the health care available locally. In less developed countries, this is one of the ways for patients to have access to new, otherwise unavailable drugs. It is agreed that due to high level of regulation of this type of research patients as participants in clinical trials should be provided with direct health benefits, under the controlled risks that are not be greater than those associated with routine medical care or a disease progression.

I came across an article written on the occasion of International Clinical Trials Day, which raises the question of whether implementation of clinical trials in the local community is needed [1]. Indeed, not a lot of research articles address the impact of large-scale clinical research on the local community, the health system itself and the quality of health care. When it comes to clinical trials, people generally agree that those are profitable for the pharmaceutical industry and researchers themselves. Less are mentioned direct investments of the sponsors (mainly through the supplied medicines, but also financing diagnostic tests etc.) that enable significant savings for hospitals and healthcare systems. The estimates of direct investments of pharmaceutical industry are calculated in millions per year. In some countries of the Western Europe, as much as 13\% of hospital budgets are made up of clinical trial revenues [1].

Health research is expected to have a direct impact on health policy and healthcare. Study of Cohen, at al. [2] focused on intervention research studies impact on healthcare policy and practice. They found measurable post-research impacts, with $38 \%$ of the studies having some impacts on policy and practice. On the other hand, it has been recognized that focus of pharmaceutical industry is not on certain clinical specialties when it is about generating information that would enable decision makers to develop therapeutic guidelines [3,4]. RCT sponsored by pharmaceutical industry are generating high quality evidence in a costly way, while the results are not fully applicable in clinical care due to the ideal setting of conduct and rigorously designed inclusion and exclusion criteria. Generated data can be very specific and hardly generalizable to the broader patient population. The governments and administration attempt to influence the direction of clinical research locally, through public funds and other sustained kinds of financial support in order to obtain data of interest. The budget of United States National Institutes of Health for this purpose was $\$ 31.2$ billion, while United Kingdom had healthcare research budget of $€ 1.7$ billion [4].

Research about RCT is usually done by those involved in their implementation, so the focus is on practical aspects of design and conduct or issues with patient enrollment. Through this kind of research, it has been 


\section{Journal of Quality in Health Care \& Economics}

recognized that providing information to the local community about the relevant findings of the RCT is important for the perception of the significance of this form of research, including the dissemination of results directly to the patients who participated in the trials as participants [5].

Within the Research Excellence Framework (REF) conducted in 2014, the United Kingdom universities and medical schools submitted 1,621 case studies in order to demonstrate the impact of their biomedical research during the period of last 20 years. Those case studies were assessed, and many were considered to provide examples of the benefits from such research. These benefits ranged from new developments of treatments to the screening programmes that yield reductions in mortality and morbidity [6]. It was suggested that research-active hospitals may offer wider treatment options on the benefit of all patients, and that hospitals engaged in the conduct of clinical trials have better health outcomes [7].

Insufficient data is available about the impact on quality improvement in healthcare itself. One of the definitions of quality improvement being "systematic, data-guided activities designed to bring about immediate, positive change in the delivery of health care in a particular setting" [8]. Majumdar, et al. [9] compared care and mortality rate for hospitals that are or are not participating in clinical trials. In conclusion authors suggested that those hospitals that do participate in trials seem to provide better care and to have lower mortality in certain indications. The caution should be applied regarding generalizing those data. Apart from other reasons, systematic differences in patient characteristics may be present depending on the hospital in which they are being treated [4]. For example, academic and research-active institutions could attract more educated and wealthy patients, these should be considered since patient population and their diseases characteristics may also impact healthcare outcomes significantly. Nevertheless, a study in Netherlands provided evidence that training, and quality assurance associated with a large study was associated with a fast improvement in national outcome statistics [10].

A systematical difference was suggested for health professionals that practice in research-active environment, because of personal characteristics, multidisciplinary collaboration, or additional training and education that is available to them. Implementation of clinical research programs seems to increase the retention of gifted clinicians and scientists [4]. Widening of opportunities for collaboration in research and participating in new developments could be very motivating. Also, there is a potential of increasing of visibility of investigators and their institutions in international medical scientists' circles, given the coauthorship or acknowledgements in published clinical trial articles.

The rationale for the potential impact of large-scale clinical trials on the quality of health care could lie primarily in the new experiences and knowledge that this type of research brings to hospitals. The available patient pool is what attracts the sponsors, while a prerequisite for participating in a large-scale clinical trial is availability of infrastructure in terms of accommodation, equipment and qualified researchers. Through participation in large-scale clinical studies, physicians are given the opportunity to apply a state-of-the-art drugs and treatment method, which may affect their future professional practice and therapeutic decisioning. It is possible that research-active institutions are more likely to follow up-to-date guidelines and introduce new evidence into practice faster [4]. It would be interesting to investigate indirect benefits of enhancement of the medical knowledge and skills of physicians participating in the studies as investigators, and whether the transfer of this new knowledge occurs within the healthcare institutions where the research is being conducted.

\section{References}

1. Bohunicki $H$, Gacic $M$, Martinovic $S$, Inicijativa za klinička ispitivanja (2016) Do Croatia need clinical trials? Medicine and science.

2. Cohen G, Schroeder J, Newson R, King L, Rychetnik L, et al. (2014) Does health intervention research have real world policy and practice impacts: testing a new impact assessment tool. Health Research Policy and Systems 13: 3 .

3. Weinfurt PK, HernandezFA, Coronado DG, DeBar LL, Dember LM, et al. (2017) Pragmatic clinical trials embedded in healthcare systems: generalizable lessons from the NIH Collaboratory. BMC Medical Research Methodology 17: 144.

4. Krzyzanowska KM, Kaplan R, Sullivan R (2011) How may clinical research improve healthcare outcomes? Annals of Oncology 22(S7): 710-715.

5. Nass JS, Levit AL, Gostin OL (2009) Beyond the HIPAA Privacy Rule: Enhancing Privacy, Improving Health through Research. Committee on Health Research and 


\section{Journal of Quality in Health Care \& Economics}

the Privacy of Health Information: The HIPAA Privacy Rule; Institute of Medicine. Washington (DC): National Academies Press (US).

6. Hanney RS, González-Block AM (2015) Health research improves healthcare: now we have the evidence and the chance to help the WHO spread such benefits globally Health Research Policy and Systems 13: 12 .

7. Young E (2015) The importance of research in healthcare. South Sudan Medical Journal 8(4).
8. Baily MA (2008) Harming through protection?. New England Journal of Medicine. 258(8):768-769.

9. Majumdar SR, Roe MT, Peterson ED, Chen AY, Gibler $\mathrm{WB}$, et al. (2008) Better Outcomes for Patients Treated at Hospitals That Participate in Clinical Trials. Arch Intern Med 168(6): 657-662.

10. Van Gijn W, Krijnen P, Lemmens VE, den Dulk M, Putter H, et al. (2010) Quality assurance in rectal cancer treatment in the Netherlands: a catch up compared to colon cancer treatment. Eur J Surg Oncol 36(4): 340-344. 